\title{
A NOTE ON THE VOLUME COMPARISON OF TUBES AROUND GEODESICS
}

\author{
JONG-GUG YUN
}

ABStRACT. In this paper, we shall calculate the volume of normal tubes around geodesics under a curvature perturbation to establish a theorem of volume comparison type.

\section{Introduction}

Let $M$ be an $n$-dimensional Riemannian manifold with a submanifold $N$ of dimension $k(<n)$. The normal tube of radius $r$ around $N$ is defined as $T(N, r)=\left\{x \in M \mid x=\exp _{N}(t v)\right.$, where $|t|<r$ and $\left.v \in \nu(N)\right\}$. Here, $\nu(N)$ is the normal bundle of $N$ in $M$ consisting of vectors perpendicular to $N$ and $\exp _{N}: \nu(N) \rightarrow M$ is the normal exponential map. Some formulas for the volume of tubes around $N$, where $N$ is a hypersurface or a point are studied in [3], [10] and a new method was found for estimating the volume of tubes around closed geodesics in [6].

When $N$ is any geodesic $\gamma:[0, a] \rightarrow M$, it was also shown in [8] that an analogue of the result of Heintz and Karcher [6] can be obtained in the situation where one has $L^{p}$-curvature bounds, which measure the quantities of the sectional curvature lying below a given number $\lambda$ in the $L^{p}$-norm. More specifically, if $f(x)$ is the smallest sectional curvature of a plane in $T_{x} M$, then we consider

$$
K(\lambda, p)=\int_{M}\{\max \{-f(x)+\lambda, 0\}\}^{p} d \mathrm{vol} .
$$

Indeed, in [8], the following theorem was obtained.

Theorem $1.1([8])$. Let $N \subset M$ be a geodesic and $\lambda \leq 0$. Then the volume of the normal tube around $N$ satisfies $\operatorname{vol} T(N, r) \leq F(n, p, a, b, c, r)$, where

$$
\begin{gathered}
a=l(N)=\text { length of } N, \\
b=|\lambda|^{p}, c=K(\lambda, p), p>n-1 .
\end{gathered}
$$

Furthermore, as $a, c \rightarrow 0$ we have that $F(n, p, a, b, c, r) \rightarrow 0$.

Received May 8, 2007; Revised January 3, 2008.

2000 Mathematics Subject Classification. 53C20.

Key words and phrases. mean curvature, sectional curvature. 
In this paper, we shall show that if the sectional curvature $K_{M}$ of $M$ is bounded below by a real number, then we obtain a comparison result analogous to the classical Bishop-Gromov volume comparison for the volumes of tubes around a geodesic in $M$ and in the standard sphere $S^{n}(1)$ with constant curvature 1 by pinching $K(1, p)$ for $p \geq 1$.

To state our main theorem more specifically, we need some notations as following. Note first that using the fact that $N$ is a geodesic we can use a parallel frame along $N$ to coordinatize the normal tubes $T(N, r)$ as $(s, t, \theta)$, where $s$ is the arclength parameter on $N, t$ measures the distance to $N$ and $\theta \in \mathrm{S}^{n-2}(1)$ is the angular parameter from the unit normal bundle. Now write the Riemannian volume element as

$$
d \mathrm{vol}=\omega(s, t, \theta) d t \wedge d s \wedge d \theta_{n-2},
$$

where $d \theta_{n-2}$ is the standard volume form on $\mathrm{S}^{n-2}(1)$. As $t$ increases $\omega$ may become undefined but we can just define it to be zero for these $t$ for our purpose to estimate the volume.

We also define $J$ by $J^{n-2}=\omega$ to obtain the initial conditions $J(0)=$ $0, J^{\prime}(0)=1$, where $/$ denotes the differentiation with respect to $t$.

In case of the space form $\mathrm{S}^{n}(\kappa)$ with constant curvature $\kappa \in \mathbb{R}$, an immediate calculation (refer to Lemma 2.2 and p. 39 in [2]) shows that the metric is given by

$$
g=C_{\kappa}^{2}(t) d s^{2}+d t^{2}+S_{\kappa}^{2}(t) d \theta_{n-2},
$$

where $C_{\kappa}(t)$ (resp. $S_{\kappa}(t)$ ) is the unique solution of the Jacobi equation for constant curvature $\kappa$, namely

$$
x^{\prime \prime}(t)+\kappa x(t)=0
$$

with initial conditions $x(0)=1, x^{\prime}(0)=0\left(\right.$ resp. $\left.x(0)=0, x^{\prime}(0)=1\right)$.

So in this case we have that

$$
\omega_{\kappa}(t)=C_{\kappa}(t) S_{\kappa}^{n-2}(t) .
$$

Furthermore, if we let $\omega^{\prime}=h \omega$, then we obtain the differential inequality as following (for details, refer to [10]).

$$
h^{\prime}+\frac{h^{2}}{n-1} \leq-\operatorname{Ric}\left(\partial_{t}, \partial_{t}\right)
$$

Here, $\partial_{t}$ is the unit gradient vector field for the distance function to $N$. We know that $h$ is the mean curvature of the level set of distance function to $N$. It is also easy to check that for the case of $\mathrm{S}^{n}(\kappa)$, we have

$$
h_{\kappa}(t)=\frac{C_{\kappa}^{\prime}(t)}{C_{\kappa}(t)}+(n-2) \frac{S_{\kappa}^{\prime}(t)}{S_{\kappa}(t)} .
$$

Now we are in a position to state our result as follows.

Theorem 1.2. Let $N$ be a given geodesic $\gamma:[0, a] \rightarrow M$ and $0<R<\frac{\pi}{2}$ be given. We also assume that $n>2, \kappa \in \mathbb{R}, 0<r<R$. Then for every 
$\bar{\epsilon}>0$, there exists a $\delta=\delta(n, \kappa, a, r, R, \bar{\epsilon})$ such that if $M$ is an $n$-dimensional Riemannian manifold with $K_{M} \geq \kappa$ and $K(1, p)<\delta,(p \geq 1)$, then we have

$$
\frac{\operatorname{vol} T(N, R)}{\operatorname{vol} \bar{T}(R)}<\frac{\operatorname{vol} T(N, z)}{\operatorname{vol} \bar{T}(z)}+\bar{\epsilon}
$$

for all $z$ with $r<z<R$, where $\operatorname{vol} \bar{T}(r)$ is the volume of a normal $r$-tube around a geodesic $\bar{\gamma}:[0, a] \rightarrow \mathrm{S}^{n}(1)$.

Remark 1.1. When compared with the similar results in [5], the curvature condition in the above theorem has been weakened to a situation where one has 'deep wells of sectional curvature' below a positive real number by considering the standard arguments for metric rescaling.

But it should still be pointed out that the above theorem holds only for $r>0$, so it is not the complete generalization of those results in [5].

\section{Preliminaries}

Note first that the smallness of $L^{1}$-norm of the sectional curvature which is bounded below implies the smallness of $L^{p}$-norm of the curvature for any $p \geq 1$. So without loss of generality, we may assume that $p=1$ in Theorem 1.2 and for this reason, throughout this section we denote by $M$ an $n$-dimensional Riemannian manifold satisfying $K(1,1)=\int_{M}\{\max \{1-f(x), 0\}\} d$ vol $<\delta$ and $K_{M} \geq \kappa$ for any real number $\kappa$ and a positive number $\delta$.

We also let $N$ and $R>0$ be given as in the previous section and define, for any $\eta>0, E_{\eta}:=\{x \in T(N, R) \mid \max \{1-f(x), 0\}>\eta\}$.

Note first that $\operatorname{vol}\left(E_{\eta}\right)$ converges to zero as $\delta \rightarrow 0$. This follows immediately since

$$
\begin{aligned}
\int_{M}\{\max \{1-f(x), 0\}\} d \mathrm{vol} & >\int_{T(N, R)}\{\max \{1-f(x), 0\}\} d \mathrm{vol} \\
& >\int_{E_{\eta}} \eta d \mathrm{vol}=\eta \operatorname{vol}\left(E_{\eta}\right) .
\end{aligned}
$$

Now we let $\mu_{s, \theta}$ be the measure on $\exp _{\gamma(s)} t \theta=: C_{s, \theta}(t)$ for each $s(0 \leq s \leq a)$, $\theta \in \mathrm{S}^{n-2}(1)$ and we define $\nu>0$ so that $\omega_{\kappa}(\nu)=\sqrt{\epsilon}$, where $\epsilon=\operatorname{vol}\left(E_{\eta}\right)$.

From now on, we proceed similar arguments as in [7] and define the explicit quantities in our case as follows.

$$
S_{\sqrt[4]{\epsilon}, \nu}(s, \theta)=\inf \left\{c \mid c>\nu,(s, \theta) \in\left(\Phi_{\sqrt[4]{\epsilon}, \nu}\right)^{c}, \mu_{s, \theta}\left(C_{s, \theta}([\nu, c]) \cap E_{\eta}\right) \geq \sqrt[4]{\epsilon}\right\},
$$

where

$$
\Phi_{\sqrt[4]{\epsilon}, \nu}=\left\{(s, \theta) \in[0, a] \times \mathrm{S}^{n-2} \mid \mu_{s, \theta}\left(C_{s, \theta}([\nu, R]) \cap E_{\eta}\right)<\sqrt[4]{\epsilon}\right\} .
$$

Then we obtain the similar lemma as in [7] which will be used later in Section 4 to estimate the volume of normal tubes.

Lemma 2.1. $\operatorname{vol}\left\{\exp _{\gamma(s)} t \theta \mid(s, \theta) \in\left(\Phi_{\sqrt[4]{\epsilon}, \nu}\right)^{c}, S_{\sqrt[4]{\epsilon}, \nu}(s, \theta) \leq t \leq R\right\}$ converges to zero as $\delta \rightarrow 0$. 
The proof of this lemma basically follows the arguments in [7]. Let first

$$
\Psi_{\sqrt[4]{\epsilon}, \nu}=\left\{(s, \theta) \in(\Phi \sqrt[4]{\epsilon, \nu})^{c} \mid \int_{C_{s, \theta}([\nu, R]) \cap E_{\eta}} \omega(s, t, \theta) d t \geq \sqrt{\epsilon}\right\} .
$$

Since

$$
\begin{aligned}
\epsilon=\operatorname{vol}\left(E_{\eta}\right) & =\int_{\mathrm{S}^{n-2} \times[0, a]}\left(\int_{C_{s, \theta}([0, R]) \cap E_{\eta}} \omega(s, t, \theta) d t\right) d s d \theta \\
& \geq \int_{\Psi_{\sqrt[4]{\epsilon}, \nu}}\left(\int_{C_{s, \theta}([\nu, R]) \cap E_{\eta}} \omega(s, t, \theta) d t\right) d s d \theta \\
& \geq \sqrt{\epsilon} \operatorname{vol}\left(\Psi_{\sqrt[4]{\epsilon}, \nu}\right)
\end{aligned}
$$

$\operatorname{vol}\left(\Psi_{\sqrt[4]{\epsilon, \nu}}\right)$ converges to zero as $\delta \rightarrow 0$.

Thus we may assume that for every $(s, \theta) \in\left(\Phi_{\sqrt[4]{\epsilon}, \nu}\right)^{c}$,

$$
\int_{C_{s, \theta}([\nu, R]) \cap E_{\eta}} \omega(s, t, \theta) d t<\sqrt{\epsilon} .
$$

We then know that there exists a $d>\nu$ such that $\omega(s, d, \theta)<\sqrt[4]{\epsilon}$ and $\mu_{s, \theta}\left(C_{s, \theta}([\nu, d]) \cap E_{\eta}\right) \leq \sqrt[4]{\epsilon}$. Of course, we know that $d \leq S_{\sqrt[4]{\epsilon}, \nu}(s, \theta)$.

Furthermore, from the condition $K_{M} \geq \kappa$, it is easy to show the following inequality using the standard arguments.

$$
\frac{\omega^{\prime}}{\omega} \leq \frac{\omega_{\kappa}^{\prime}}{\omega_{\kappa}}
$$

Thus we have for any $t$ with $S_{\sqrt[4]{\epsilon}, \nu}(s, \theta) \leq t \leq R$ and $(s, \theta) \in\left(\Phi_{\sqrt[4]{\epsilon}, \nu}\right)^{c}$,

$$
\begin{aligned}
\omega(s, t, \theta) & \leq \frac{\omega_{\kappa}(t)}{\omega_{\kappa}(d)} \omega(s, d, \theta) \\
& \leq \frac{\max \left\{\omega_{\kappa}(r) \mid 0 \leq r \leq R\right\}}{\omega_{\kappa}(\nu)} \sqrt[4]{\epsilon} \\
& =\max \left\{\omega_{\kappa}(r) \mid 0 \leq r \leq R\right\} \sqrt[8]{\epsilon},
\end{aligned}
$$

which converges to zero as $\delta \rightarrow 0$. This completes our proof.

\section{Mean curvature comparison}

In this section, we compare the mean curvature $h(s, t, \theta)$ in $M$ with $h_{1}(t)$ in $\mathrm{S}^{n}(1)$. Recall that we have two assumptions on the curvature of $M$ as follows:

$$
K_{M} \geq \kappa, \int_{M}\{\max \{1-f(x), 0\}\} d \mathrm{vol}<\delta
$$

for any real number $\kappa$ and a positive number $\delta$.

We will use the same notations as in the previous section and provide the comparison theorem as following.

Theorem 3.1. For any $(s, \theta) \in \Phi_{\sqrt[4]{\epsilon}, \nu}$, we have $h(s, r, \theta) \leq h_{1}(r)+\tau(\epsilon)$ for all $r, R$ with $0 \leq r \leq R<\frac{\pi}{2}$, where $\tau(\epsilon) \rightarrow 0$ as $\epsilon \rightarrow 0$. 
We first take an orthonormal parallel vector fields $\left\{E_{i}(t)\right\}_{i=1}^{n}$ along $C_{s, \theta}(t)$ such that $E_{1}(0)=C_{s, \theta}^{\prime}(0), E_{2}(0)=\gamma^{\prime}(s)$.

Now we recall that by the standard theory of index form we have the following.

$$
\begin{aligned}
h(s, r, \theta) \leq & \int_{0}^{r}\left\{\left(\frac{C^{\prime}(t)}{C(r)}\right)^{2}-\left(\frac{C(t)}{C(r)}\right)^{2} K\left(E_{2}, C_{s, \theta}^{\prime}(t)\right)\right\} d t-\left[\frac{C(t)}{C(r)} \cdot \frac{C^{\prime}(t)}{C(r)}\right]_{0}^{r} \\
& +\sum_{i=3}^{n} \int_{0}^{r}\left\{\left(\frac{S^{\prime}(t)}{S(r)}\right)^{2}-\left(\frac{S(t)}{S(r)}\right)^{2} K\left(E_{i}, C_{s, \theta}^{\prime}(t)\right)\right\} d t \\
& -(n-2)\left[\frac{S(t)}{S(r)} \cdot \frac{S^{\prime}(t)}{S(r)}\right]_{0}^{r},
\end{aligned}
$$

where $S(t)=\frac{1}{\sqrt{1-\eta}} \sin (\sqrt{1-\eta} t), C(t)=\cos (\sqrt{1-\eta} t)$.

Note that the right hand side of the above inequality can be rewritten as follows (refer to p. 142 in [4]).

$$
\begin{aligned}
& \int_{0}^{r}\left(\frac{C^{\prime}(t)}{C(r)}\right)^{2}\left((1-\eta)-K\left(E_{2}, C_{s, \theta}^{\prime}(t)\right)\right) d t \\
& +\sum_{i=3}^{n} \int_{0}^{r}\left(\frac{S^{\prime}(t)}{S(r)}\right)^{2}\left((1-\eta)-K\left(E_{i}, C_{s, \theta}^{\prime}(t)\right)\right) d t \\
& +\left[\frac{C(t)}{C(r)} \cdot \frac{C^{\prime}(t)}{C(r)}\right]_{0}^{r}+(n-2)\left[\frac{S(t)}{S(r)} \cdot \frac{S^{\prime}(t)}{S(r)}\right]_{0}^{r}
\end{aligned}
$$

We first observe that the third and fourth terms in the above sum turn into $h_{1-\eta}(r)=(n-2) \sqrt{1-\eta} \cot (\sqrt{1-\eta} r)-\sqrt{1-\eta} \tan (\sqrt{1-\eta} r)$.

By letting $\eta$ as small as we please, we can express this as $h_{1}(r)+\tau(\eta)(r<$ $R<\frac{\pi}{2}$ ), where $\tau(\eta)$ converges to zero as $\eta \rightarrow 0$.

Next, in the first and second terms in the above sum, we break the interval $[0, r]$ of integration into three parts as follows.

$$
C_{s, \theta}([0, r])=C_{s, \theta}([0, \nu]) \cup\left\{C_{s, \theta}([\nu, r]) \cap E_{\eta}\right\} \cup\left\{C_{s, \theta}([\nu, r]) \cap E_{\eta}^{c}\right\} .
$$

First of all, note that $(1-\eta)-K\left(E_{i}, C_{s, \theta}^{\prime}(t)\right) \leq 0$ on $C_{s, \theta}([\nu, r]) \cap E_{\eta}^{c}$ for $i=2, \ldots, n$. So the integration in this part is negative.

On $C_{s, \theta}([\nu, r]) \cap E_{\eta}$ on the other hand, we have $(1-\eta)-K\left(E_{i}, C_{s, \theta}^{\prime}(t)\right) \leq$ $1-\eta-\kappa$ and $\mu_{s, \theta}\left(C_{s, \theta}([\nu, R]) \cap E_{\eta}\right)<\sqrt[4]{\epsilon}$. Thus we can say that

$$
\begin{aligned}
& \int_{C_{s, \theta}([\nu, R]) \cap E_{\eta}}\left(\frac{C^{\prime}(t)}{C(r)}\right)^{2}\left((1-\eta)-K\left(E_{2}, C_{s, \theta}^{\prime}(t)\right)\right) d t \\
& +\sum_{i=3}^{n} \int_{C_{s, \theta}([\nu, R]) \cap E_{\eta}}\left(\frac{S^{\prime}(t)}{S(r)}\right)^{2}\left((1-\eta)-K\left(E_{i}, C_{s, \theta}^{\prime}(t)\right)\right) d t \leq \tau(\epsilon),
\end{aligned}
$$

where $\tau(\epsilon) \rightarrow 0$ as $\epsilon \rightarrow 0$. 
Finally on $C_{s, \theta}([0, \nu])$, we break it into $C_{s, \theta}([0, \nu]) \cap E_{\eta}$ and $C_{s, \theta}([0, \nu]) \cap E_{\eta}^{c}$ and apply the same arguments as on $C_{s, \theta}([\nu, r]) \cap E_{\eta}$ and $C_{s, \theta}([\nu, r]) \cap E_{\eta}^{c}$ to obtain the similar estimates noting that $\nu$ can be chosen arbitrarily small as $\epsilon \rightarrow 0$. Consequently, by putting all these estimates together, we conclude that $h(r, s, \theta) \leq h(r)+\tau(\epsilon)$, which completes the proof.

\section{Volume comparison}

We finally estimate the volume of the normal tube around a geodesic in our case and obtain a comparison theorem which is analogous to the classical Bishop-Gromov volume comparison. Every notations in this section also follow those in Section 2.

Recall first that we declared $\omega(s, t, \theta)$ to be zero whenever it is undefined since $t$ goes too far from $N$.

Now let us analyze the volume of $T(N, R)$ as following.

$$
\begin{aligned}
& \operatorname{vol} T(N, R)=\int_{\Phi \sqrt[4]{\epsilon}, \nu}\left(\int_{0}^{R} \omega(s, t, \theta) d t\right) d s d \theta_{n-2} \\
& +\int_{\left(\Phi_{\sqrt[4]{\epsilon}, \nu}\right)^{c}}\left(\int_{0}^{S \sqrt[4]{\epsilon}, \nu}(\theta, s) \omega(s, t, \theta) d t\right) d s d \theta_{n-2} \\
& +\int_{\left(\Phi_{\sqrt[4]{\epsilon, \nu})^{c}}\right.}\left(\int_{S_{\sqrt[4]{\epsilon, \nu}}(\theta, s)}^{R} \omega(s, t, \theta) d t\right) d s d \theta_{n-2} .
\end{aligned}
$$

Here, note that the last term above can be written as $\xi(\delta)$, which converges to 0 as $\delta \rightarrow 0$ by Lemma 2.1.

By the same arguments as in the proof of Theorem 3.1, we can say that on $\Psi:=\Psi_{1} \cup \Psi_{2}$, where

$$
\Psi_{1}=\{(s, t, \theta) \mid(s, \theta) \in \Phi \sqrt[4]{\epsilon, \nu}, 0 \leq t \leq R\}
$$

and

$$
\Psi_{2}=\left\{(s, t, \theta) \mid(s, \theta) \in\left(\Phi_{\sqrt[4]{\epsilon}, \nu}\right)^{c}, 0 \leq t \leq S_{\sqrt[4]{\epsilon}, \nu}(\theta, s)\right\}
$$

we have

$$
h(s, t, \theta)-h_{1}(t)<\tau(\epsilon)
$$

for some $\tau(\epsilon)>0$ where $\tau(\epsilon)$ can be arbitrarily small as $\epsilon \rightarrow 0$.

From this fact, a straightforward calculation of integration shows that

$$
\frac{\omega\left(s, t_{2}, \theta\right)}{\omega_{1}\left(t_{2}\right)}<\exp (\alpha(\epsilon)) \frac{\omega\left(s, t_{1}, \theta\right)}{\omega_{1}\left(t_{1}\right)}
$$

for any $\left(s, t_{1}, \theta\right),\left(s, t_{2}, \theta\right) \in \Psi$ with $t_{1}<t_{2}$, where $\alpha(\epsilon)$ converges to zero as $\epsilon \rightarrow 0$. Now we reproduce Lemma 2.1 in [9] to proceed our arguments and just sketch the proof below. 
Lemma 4.1. Let $f, g$ be two positive continuous functions defined on $[0, \infty]$. If $\frac{f(b)}{g(b)} \leq \exp (\alpha) \frac{f(a)}{g(a)}$ for some $\alpha>0$ and for all $a, b$ with $0<a<b$, then for any given $R>0, r>0$ and with $R>r>0$ we have

$$
\frac{\int_{0}^{R} f(t) d t}{\int_{0}^{R} g(t) d t} \leq \frac{\int_{0}^{z} f(t) d t}{\int_{0}^{z} g(t) d t}+\tau(\alpha)
$$

for all $z>0$ with $R \geq z \geq r>0$ and for some $\tau(\alpha)>0$ satisfying $\lim _{\alpha \rightarrow 0} \tau(\alpha)=$ 0 .

It suffices to show that the function $F(y)=\frac{\int_{0}^{y} f(t) d t}{\int_{0}^{y} g(t) d t}$ is almost nonincreasing with respect to $y \in[r, R]$.

Specifically, we compute

$$
F^{\prime}(y) \leq \frac{g(y) \int_{0}^{y} g(t) d t}{\left(\int_{0}^{y} g(t) d t\right)^{2}} \frac{\int_{0}^{y} f(t) d t}{\int_{0}^{y} g(t) d t}(\exp (\alpha)-1)
$$

for all $y$ with $r \leq y \leq R$.

Since the right hand of the above inequality tends to zero as $\alpha \rightarrow 0$, we can express $F^{\prime}(y) \leq \mu(\alpha)$ for some $\mu(\alpha)>0$ satisfying $\lim _{\alpha \rightarrow 0} \mu(\alpha)=0$. Then by integrating this inequality from $z$ to $R$, we get $F(R)-F(z) \leq(R-z) \mu(\alpha)$. So if we let $\tau(\alpha):=(R-z) \mu(\alpha)<R \mu(\alpha)$, then we have $F(R) \leq F(z)+\tau(\alpha)$, which is our desired result.

For the $\alpha=\alpha(\epsilon)$ in (4.2), we define $y_{0}$ so that $\int_{0}^{y_{0}} \omega_{1}(t) d t=\sqrt{\alpha}$. Then from (4.3) in the proof of the above Lemma 4.1 and (4.2), it is easy to check

$$
\left.\left(\frac{\int_{0}^{y} \omega d t}{\int_{0}^{y} \omega_{1} d t}\right)^{\prime}\right|_{y_{0} \leq y \leq R} \leq \frac{\exp (\alpha)-1}{\sqrt{\alpha}} C(k, n, R),
$$

which converges to zero as $\alpha \rightarrow 0$. So we have

$$
\frac{\int_{0}^{R} \omega d t}{\int_{0}^{R} \omega_{1} d t} \leq \frac{\int_{0}^{z} \omega d t}{\int_{0}^{z} \omega_{1} d t}+\tau(\alpha(\epsilon))
$$

for all $z$ with $y_{0} \leq z \leq R$, where $\tau(\alpha(\epsilon))>0$ goes to zero as $\epsilon \rightarrow 0$.

From the above inequality, we can easily obtain the following.

$$
\frac{\int_{\Phi \sqrt[4]{\epsilon, \nu}}\left(\int_{0}^{R} \omega d t\right) d s d \theta_{n-2}}{\int_{\mathrm{S}^{n-2}(1) \times[0, a]}\left(\int_{0}^{R} \omega_{1} d t\right) d s d \theta_{n-2}} \leq \frac{\int_{\Phi_{\sqrt[4]{\epsilon}, \nu}}\left(\int_{0}^{z} \omega d t\right) d s d \theta_{n-2}}{\int_{\mathrm{S}^{n-2}(1) \times[0, a]}\left(\int_{0}^{z} \omega_{1} d t\right) d s d \theta_{n-2}}+\tau(\alpha(\epsilon))
$$

for all $z$ with $y_{0} \leq z \leq R$.

Next, we shall estimate the volume ratio for the case $(s, t, \theta) \in \Psi_{2}$ in the similar way. Note first that $\left(\Phi_{\sqrt[4]{\epsilon}, \nu}\right)^{c}$ can be divided into the following three subsets:

$$
\left(\Phi_{\sqrt[4]{\epsilon}, \nu}^{1}\right)^{c}=\left\{(s, \theta) \in\left(\Phi_{\sqrt[4]{\epsilon}, \nu}\right)^{c} \mid S_{\sqrt[4]{\epsilon}, \nu}(s, \theta)<y_{0}<R\right\}
$$




$$
\left(\Phi_{\sqrt[4]{\epsilon}, \nu}^{2}\right)^{c}=\left\{(s, \theta) \in\left(\Phi_{\sqrt[4]{\epsilon}, \nu}\right)^{c} \mid y_{0}<S_{\sqrt[4]{\epsilon}, \nu}(s, \theta)<R\right\}
$$

and

$$
\left(\Phi_{\sqrt[4]{\epsilon}, \nu}^{3}\right)^{c}=\left\{(s, \theta) \in\left(\Phi_{\sqrt[4]{\epsilon}, \nu}\right)^{c} \mid y_{0}<R<S_{\sqrt[4]{\epsilon}, \nu}(s, \theta)\right\} .
$$

For the case $(s, t, \theta) \in \Psi_{2}$ and $(s, \theta) \in\left(\Phi_{\sqrt[4]{\epsilon}, \nu}^{1}\right)^{c}$, we get obviously for all $z$ with $y_{0} \leq z \leq R$ that

$$
\frac{\int_{\left(\Phi_{\sqrt[4]{\epsilon}, \nu}^{1}\right)^{c}}\left(\int_{0}^{S} \int_{\sqrt[4]{\epsilon, \nu}}(s, \theta) \omega d t\right) d s d \theta_{n-2}}{\int_{\mathrm{S}^{n-2}(1) \times[0, a]}\left(\int_{0}^{R} \omega_{1} d t\right) d s d \theta_{n-2}} \leq \frac{\int_{\left(\Phi_{\sqrt[4]{\epsilon}, \nu}^{1}\right) c}\left(\int_{0}^{z} \omega d t\right) d s d \theta_{n-2}}{\int_{\mathrm{S}^{n-2}(1) \times[0, a]}\left(\int_{0}^{z} \omega_{1} d t\right) d s d \theta_{n-2}} .
$$

For the case $(s, t, \theta) \in \Psi_{2}$ and $(s, \theta) \in\left(\Phi_{\sqrt[4]{\epsilon, \nu}}^{2}\right)^{c}$, we use Lemma 4.1 and obtain

$$
\begin{aligned}
& \frac{\int_{\left(\Phi_{\sqrt[4]{\epsilon}, \nu}^{2}\right)^{c}}\left(\int_{0}^{S} S_{\sqrt[4]{\epsilon}, \nu}(s, \theta) \omega d t\right) d s d \theta_{n-2}}{\int_{\mathrm{S}^{n-2}(1) \times[0, a]}\left(\int_{0}^{R} \omega_{1} d t\right) d s d \theta_{n-2}} \\
\leq & \frac{\int_{\left(\Phi_{\sqrt[4]{\epsilon}, \nu}^{2}\right)^{c}}\left(\int_{0}^{z} \omega d t\right) d s d \theta_{n-2}}{\int_{\mathrm{S}^{n-2}(1) \times[0, a]}\left(\int_{0}^{z} \omega_{1} d t\right) d s d \theta_{n-2}}+\tau(\alpha(\epsilon))
\end{aligned}
$$

for all $z$ with $y_{0} \leq z \leq S_{\sqrt[4]{\epsilon}, \nu}(s, \theta)$.

Furthermore, in case $S_{\sqrt[4]{\epsilon}, \nu}(s, \theta)<z \leq R$, we clearly have

$$
\frac{\int_{\left(\Phi_{\sqrt[4]{\epsilon}, \nu}^{2}\right)^{c}}\left(\int_{0}^{S_{\sqrt[4]{\epsilon}, \nu}(s, \theta)} \omega d t\right) d s d \theta_{n-2}}{\int_{\mathrm{S}^{n-2}(1) \times[0, a]}\left(\int_{0}^{R} \omega_{1} d t\right) d s d \theta_{n-2}} \leq \frac{\int_{\left(\Phi_{\sqrt[4]{\epsilon}, \nu}^{2}\right)^{c}}\left(\int_{0}^{z} \omega d t\right) d s d \theta_{n-2}}{\int_{\mathrm{S}^{n-2}(1) \times[0, a]}\left(\int_{0}^{z} \omega_{1} d t\right) d s d \theta_{n-2}} .
$$

So we may say that (4.6) holds for any $z$ with $y_{0} \leq z \leq R$.

Finally, we obtain the similar estimate for the case $(s, t, \theta) \in \Psi_{2}$ and $(s, \theta) \in$ $\left(\Phi_{\sqrt[4]{\epsilon, \nu}}^{3}\right)^{c}$ using the same method as above. That is, we have

$$
\frac{\int_{\left(\Phi_{\sqrt[4]{\epsilon}, \nu}^{3}\right)^{c}}\left(\int_{0}^{R} \omega d t\right) d s d \theta_{n-2}}{\int_{\mathrm{S}^{n-2}(1) \times[0, a]}\left(\int_{0}^{R} \omega_{1} d t\right) d s d \theta_{n-2}} \leq \frac{\int_{\left(\Phi_{\sqrt[4]{\epsilon}, \nu}^{3}\right)^{c}}\left(\int_{0}^{z} \omega d t\right) d s d \theta_{n-2}}{\int_{\mathrm{S}^{n-2}(1) \times[0, a]}\left(\int_{0}^{z} \omega_{1} d t\right) d s d \theta_{n-2}}+\tau(\alpha(\epsilon))
$$

for any $z$ with $y_{0} \leq z \leq R$.

Now we put together all the above four inequalities (4.4)-(4.7) and recall the analysis of $\operatorname{vol} T(N, R)$ in (4.1), which gives the following inequality:

$$
\frac{\operatorname{vol} T(N, R)-\xi(\delta)}{\operatorname{vol} \bar{T}(R)} \leq \frac{\operatorname{vol} T(N, z)}{\operatorname{vol} \bar{T}(z)}+\tau(\alpha(\epsilon)) .
$$

If we choose a sufficiently small $\delta>0$ so that $\frac{\xi(\delta)}{\operatorname{vol} \bar{T}(R)}$ can be as small as we please, (consequently, $\delta$ depends on $a$ and $R$ ) then we can say that for every 
$\bar{\epsilon}>0$, there exists a $\delta>0$ such that

$$
\frac{\operatorname{vol} T(N, R)}{\operatorname{vol} \bar{T}(R)} \leq \frac{\operatorname{vol} T(N, z)}{\operatorname{vol} \bar{T}(z)}+\bar{\epsilon}
$$

for all $z$ with $y_{0} \leq z \leq R$. Since we can adjust $y_{0}$ so that $y_{0}<r$ by requiring $\delta$ to be sufficiently small enough, we complete the proof of Theorem 1.2.

\section{References}

[1] R. Bishop, A relation between volume, mean curvature and diameter, Notices Amer. Math. Soc. 10 (1963), 364.

[2] I. Chavel, Eigen Values in Riemannian Geometry, Academic press, 1984.

[3] S. Gallot, Isoperimetric inequalities based on integral norms of Ricci curvature, Asterisque 18 (1983), 191-216.

[4] S. Gallot, D. Hullin, and J. Lafontain, Riemannian Geometry, Springer-Verlag, 1980.

[5] A. Gray, Tubes, Birkhauser Verlag, 2004.

[6] E. Heintze and H. Karcher, A general comparison theorem with applications to volume estimates for submanifolds, Ann. Sci. Ecol. Norm. Sup. 11 (1978), 451-470.

[7] S.-H. Paeng, A sphere theorem under a curvature perturbation II, Kyushu J. Math. 52 (1998), 439-454.

[8] P. Petersen, S. Shteingold, and G. Wei, Comparison geometry with integral curvature bounds, Geom. Funct. Anal. 7 (1997), 1011-1030.

[9] J.-G. Yun, Mean curvature comparison with $L^{1}$-norms of Ricci curvature, Canad. Math. Bull. 49 (2006), no. 1, 152,-160.

[10] D. Yang, Convergence of Riemannain manifolds with Integral bounds on curvature I, Ann. Sci. Ecol. Norm. Sup. 25 (1992), 77-105.

Department of Mathematics Education Korea National University of Education

Chungbuk 363-791, Korea

E-mail address: jgyun69@knue.ac.kr 